\title{
Sialoadenitis aguda secundaria al medio de contraste yodado
}

\section{Iodinated contrast-induced acute sialadenitis}

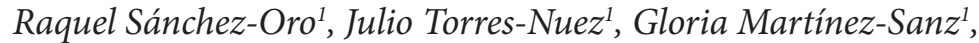 \\ María Luiza Fatahi-Bandpey ${ }^{1}$, Mohamedfadel Bleila ${ }^{1}$
}

\begin{abstract}
Resumen
La sialoadenitis es una inflamación o infección de las glándulas salivares que puede afectar a las glándulas parótidas, submandibulares y/o a las glándulas salivares menores. La sialoadenitis aguda tras la inyección de contraste yodado es una reacción adversa tardía rara, se caracteriza por un aumento de tamaño de las glándulas salivares, más frecuentemente las submandibulares. El número de estudios de tomografía computarizada ha aumentado exponencialmente en los últimos años por lo que es de esperar que cada vez se den más casos de esta entidad. El $98 \%$ del yodo inyectado por vía intravenosa es eliminado por los riñones y el $2 \%$ por otros órganos como glándulas salivales, lacrimales y sudoríparas. En los pacientes con insuficiencia renal el retraso de la eliminación renal conlleva un mayor acúmulo de yodo en la saliva que produce, a su vez, mayor inflamación de la mucosa de los conductos glandulares. Por ello, la insuficiencia renal se considera un factor de riesgo para sialoadenitis por yodo y se ha de sospechar especialmente en estos pacientes si presentan clínica sugestiva. Presentamos el caso de un paciente que tras la realización de una tomografía computarizada con contraste yodado desarrolló una sialoadenitis aguda como reacción adversa al mismo.

Palabras clave: yodo, contraste, sialoadenitis, paperas, reacción adversa.
\end{abstract}

\begin{abstract}
Sialadenitis is an inflammation or infection of the salivary glands that may affect the parotid, submandibular and/or small salivary glands. Acute sialadenitis after injection of iodinated contrast is a rare late adverse reaction. It is characterized by an enlargement of the salivary glands, most frequently the submandibular ones. The number of studies of computed tomography has increased exponentially in recent years, so it is expected that there will be more and more cases of this entity. About $98 \%$ of the iodine contrast injected intravenously is eliminated by the kidneys and $2 \%$ by other organs such as salivary, lacrimal and sweat glands. In patients with renal failure, delayed renal elimination leads to a greater accumulation of iodine in saliva, which produces greater inflammation of the mucosa of the glandular ducts. Therefore, kidney failure is considered a risk factor for iodine sialadenitis and it should be especially suspected in these patients if they present suggestive symptoms. We present a case of a patient who after performing a computed tomography study with intravenous iodinated contrast, developed acute sialadenitis as an adverse reaction to this contrast.
\end{abstract}

Keywords: Iodide, contrast, sialadenitis, mumps, adverse reaction.
'Servicio de Radiodiagnóstico, Hospital General de Teruel Obispo Polanco. Teruel, España.

Los autores declaran no tener conflictos de interés.

Recibido el 10 de abril de 2020. Aceptado el 26 de junio de 2020.

\section{Correspondencia:}

Raquel Sánchez-Oro Hospital Obispo Polanco, atención de Raquel Sánchez Oro y Servicio de Radiodiagnóstico. Avda. Ruiz Jarabo, s/n 44002. Teruel, España. Email: raquel_sanchez_oro@ hotmail.com

\section{Caso Clínico}

Varón de 64 años, con antecedentes de insuficiencia renal crónica en diálisis peritoneal, presentó un episodio de melena por lo que se le realizó una tomografía computarizada multidetector (TCMD) toraco-abdómino-pélvica tras la administración intravenosa de contraste yodado (130 mL de contraste Ultravist). No se observó sangrado activo en dicho estudio. Trascurridas 24 horas de la realización de la TCMD, el paciente acudió al servicio de Urgencias por aparición, de forma brusca, de inflamación submandibular y de la vertiente 
anterior del cuello. El paciente no asociaba fiebre, dificultad respiratoria ni otras manifestaciones clínico-analíticas de cuadro infeccioso.

Se solicitó nueva TCMD, en este caso de la región cervical, que se practicó sin administrar contraste intravenoso. En este estudio se observó un marcado aumento de tamaño ambas glándulas submandibulares (Figura 1), con tejido celular subcutáneo y tejido graso del espacio cervical anterior trabeculares, por cambios inflamatorios, así como engrosamiento del músculo platisma.

No se objetivó dilatación de los conductos excretores submandibulares (de Wharton) ni imágenes de sialolitiasis. En la reconstrucción volumétrica a partir de la TCMD (Figura 2) se apreció el aumento del volumen submandibular bilateral y de la región cervical anterior.

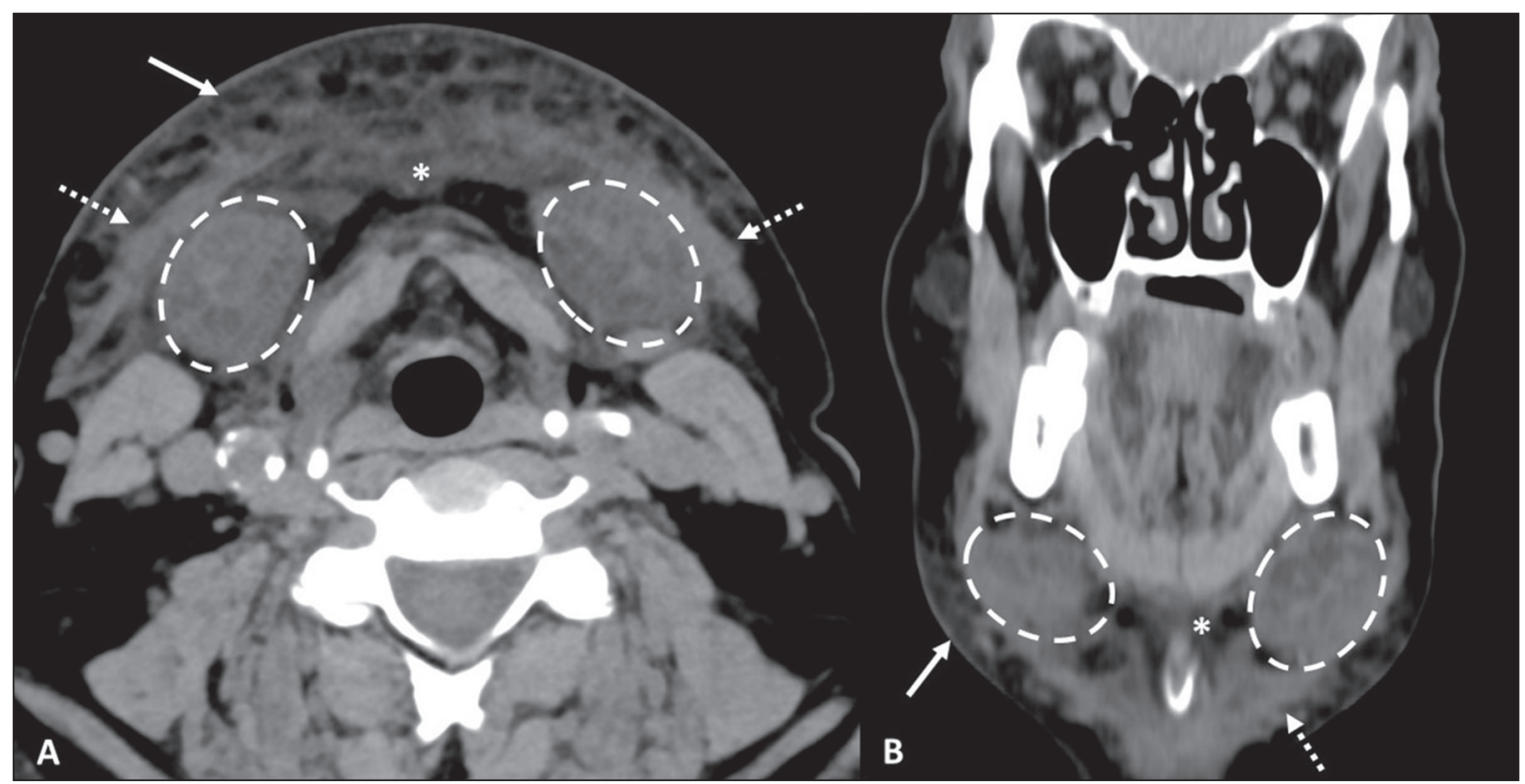

Figura 1. A: Imagen de tomografía computarizada multidetector (TCMD) cervical axial. B: Imagen de TCMD cervical reconstrucción coronal. Se observa un marcado aumento de tamaño de ambas glándulas submandibulares (óvalos discontinuos), con tejido celular subcutáneo (flechas continuas) y del tejido graso del espacio cervical anterior (asteriscos) trabeculares por cambios inflamatorios, así como engrosamiento del músculo platisma (flechas discontinuas).

Figura 2. A: Reconstrucción volumétrica 3D vista coronal del examen TCMD. B: Reconstrucción volumétrica 3D vista sagital. Se observa el aumento del volumen de la región cervical anterior.

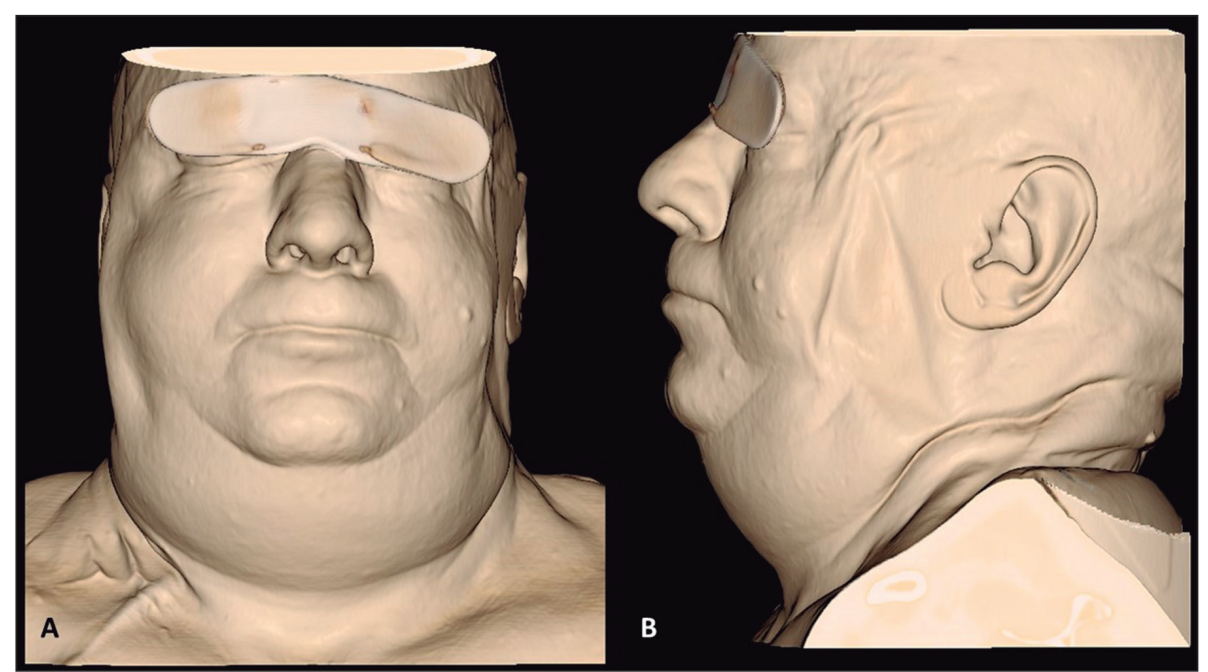


Por tanto, los hallazgos de la TCMD junto al antecedente de administración de contraste yodado 24 horas antes sugirieron el diagnóstico de sialoadenitis aguda secundaria al contraste yodado. Se administró tratamiento sintomático y el paciente presentó buena evolución con resolución de la inflamación a los tres días del inicio del cuadro clínico. Los autores disponemos de la autorización del paciente y del Comité de Ética de nuestra institución, según la normativa vigente, para presentar este trabajo.

\section{Discusión}

La inflamación de las glándulas salivares tras la administración de contraste yodado para los estudios de TCMD se considera una reacción adversa rara. Esta inflamación se conoce en la bibliografía inglesa como "paperas por yodo" ("iodide mumps")․ El primer caso de sialoadenitis por yodo fue descrito por Sussman y Miller en 1956 tras un estudio de urografía intravenosa ${ }^{1}$. De acuerdo al metaanálisis reciente de Jiao y colaboradores, el número de pacientes con dicha complicación que se ha comunicado en artículos de casos clínicos y series de casos ascendería hasta 77 casos $^{2}$. Sin embargo, hay estudios que sugieren que la sialoadenitis por yodo puede estar infradiagnosticada, con una incidencia real de entre el $1 \%$ y el $2 \%{ }^{3}$. Se ha descrito esta entidad tras la administración de contraste yodado tanto por vía oral como por la intravenosa y arterial. No se ha demostrado el mecanismo etiopatogénico concreto, aunque no parece tratarse de una reacción alérgica ${ }^{4,8}$.

Las manifestaciones clínicas de la sialoadenitis por yodo no difieren de las sialoadenitis infecciosas, litiásica u obstructiva por cuerpos extraños, traumática, o aquellas presentadas en enfermedades inflamatorias sistémicas o tumoral, siendo las manifestaciones más frecuentes el aumento de tamaño de las glándulas salivares afectadas, el dolor, la ageusia y la xerostomía ${ }^{9}$ Según el metaanálisis, comentado previamente, de Jiao y colaboradores, la edad media de los pacientes afectados es de 63 años, siendo discretamente más frecuente en hombres (61\%) que en mujeres. La sialoadenitis se desarrolla unas 16 horas de media después de la administración del contraste yodado, por esto se considera una reacción adversa tardía, a diferencia de las reacciones anafilácticas al contraste que ocurren segundos después de la administración del contraste. Aunque se han descrito casos que se desarrollan desde inmediatamente después de su administración hasta cinco días más tarde. La inflamación frecuentemente es bilateral, presentándose en $86 \%$ de los casos, una vez que se desencadena el proceso inflamatorio el aumento de volumen de las glándulas salivares se desarrolla rápidamente. La submandibular es la glándula salivar más frecuentemente afectada de forma solitaria, esto es así en el 48\% de los casos; en un $27 \%$ de los casos se afecta exclusivamente la parótida y en el 25\% están comprometidas las dos glándulas. El tratamiento esteroideo o la profilaxis con antihistamínicos no han demostrado beneficios, estando indicado solamente tratamiento sintomático (analgésicos) si existe dolor. La inflamación de las glándulas salivares se resuelve en una media de 3 días sin identificar diferencias significativas entre los pacientes que han recibido tratamiento sintomático y los que no lo han tenido ${ }^{1,2,8}$. En el caso del paciente que presentamos, recibió tratamiento analgésico con antiinflamatorios no esteroideos y tuvo buena evolución con resolución completa de los síntomas.

La edad avanzada y un mayor tiempo de evolución hasta la aparición de los síntomas, tras la administración del contraste yodado, se ha relacionado, a su vez, con retraso en la resolución de la inflamación ${ }^{2,8}$. El 98\% del contraste yodado se elimina por los riñones y el $2 \%$ restante por las glándulas salivares, lacrimales y sudoríparas. La concentración de yodo en las glándulas salivares es 100 veces superior a la plasmática. En los pacientes con insuficiencia renal el retraso en la excreción urinaria provoca un acúmulo de yodo en sangre y secundariamente en la saliva, lo que a su vez conlleva inflamación de la mucosa glandular y alteración en la secreción de la saliva, es por ello, que la insuficiencia renal se considera un factor de riesgo para desarrollar sialoadenitis por yodo ${ }^{2,4-8}$, como era el caso del paciente presentado.

Las pruebas de imagen que van a permitir realizar un diagnóstico de sialoadenitis son la ecografía cervical y la TCMD. En ecografía se identifica un aumento de volumen de las 
glándulas afectadas, con incremento de la vascularización en el estudio Doppler color y con ductos prominentes, que se muestran como estructuras hipoecoicas de morfología tubular sin señal Doppler en su interior, a diferencia de los vasos. En la TCMD se identifica también el aumento de volumen de las glándulas salivares, con trabeculado de la grasa adyacente y del tejido celular subcutáneo por cambios inflamatorios asociados. En el caso que se realice el estudio con contraste, se puede observar el incremento del realce por aumento de la vascularización. La TCMD permite excluir con mayor seguridad la presencia de sialolitiasis respecto a la ecografía. Los mismos cambios se podrían observar en un estudio de resonancia magnética, si bien al tener esta técnica menor disponibilidad para realizar estudios urgentes, se practica excepcionalmente $e^{2,4,9,10}$.

La recurrencia de esta complicación, si al paciente se le realizan nuevos estudios de TC con contraste yodado, no es infrecuente. Se ha observado que es ineficaz administrar premedicación para intentar evitar futuros episodios de sialoadenitis. Pese a esto, como se trata de una complicación autolimitada y con buen pronóstico, no debe ser un motivo para no administrar dicho contraste si se considera beneficioso para el paciente ${ }^{1,2}$.

\section{Conclusión}

La sialoadenitis aguda es una reacción poco frecuente tras la administración de contraste yodado. No se conoce su incidencia exacta, se calcula que es una complicación infradiagnosticada por el retraso en la aparición de los síntomas, su curso autolimitado y la falta de familiaridad de los médicos con esta entidad. Además, dado el aumento creciente año tras año del número de estudios de TCMD practicados, es de esperar un paulatino aumento de la incidencia. La edad avanzada y un mayor tiempo de evolución hasta la aparición de los síntomas se ha asociado con mayor duración de la sintomatología. El tratamiento esteroideo o la profilaxis con antihistamínicos no han demostrado beneficios, estando indicado solamente tratamiento analgésico si existe dolor. La recurrencia de la sintomatología no es infrecuente si se precisa realizar a los pacientes nuevos estudios de TCMD con contraste yodado, no considerándose esta complicación un motivo para no administrar dicho contraste si se considera beneficioso para el paciente ${ }^{1,2}$.

\section{Bibliografía}

1. Lucarelli, Perandini S, Borsato A, Strazimiri E, Montemezzi S. Iodinated contrast-induced sialadenitis: a review of the literature and sonographic findings in a clinical case. J Ultrason. 2018;18:359-364.

2. Jiao A, Farsad K, McVinnie DW, Jahangiri Y, Morrison JJ. Characterization of iodide-induced sialadenitis: Meta-analysis of the published case reports in the medical literature. Acad Radiol. 2020;27(3):428-435.

3. Egan M, Maglione PJ. Multiple reasonably tolerated percutaneous coronary interventions in a patient with iodide mumps. Ann Allergy Asthma Inmunol. 2015;115:253-254.

4. Sánchez S, Rubio D, Terán L, Calvo J. Sialoadenitis aguda bilateral como reacción adversa a contraste yodado. Radiología. 2018;60(2):171-174.

5. Ghosh RK, Somasundaram M, Ravakhah K. Iodide mumps following fistulogram in a haemodialysis patient. BMJ Case Rep. 2016;2:2016.

6. Afshar M, Alhussein M. Iodide-associated sialadenitis. N Engl J Med. 2017;376:868.

7. Elder $\mathrm{AM}, \mathrm{Ng} \mathrm{MK}$. Iodide mumps complicating coronary and carotid angiography. Heart Lung Circ. 2017;26:e14-e15.

8. Zhang G, Li Y, Zhang R, Guo Y, Ma Z, Wang H, et al. Acute submandibular swelling complicating arteriography with iodide contrast. Medicine. 2015;94(33): e1380.

9. Abdel Razek AAK, Mukherji S. Imaging of sialadenitis. Neuroradiol J. 2017;30(3):205-215.

10. Federici M, Guarna T, Manzi M, Della Longa G, Di Renzi P, Bellelli A. Swelling of the submandibular glands after administration of low-osmolarity contrast agent: Ultrasound findings. J Ultrasound. 2008;11(3):85-88. 